\title{
Vulvar Melanosis: Two Case Report
}

\author{
R Giménez-García ${ }^{1 *}$, Cintia Gonzalez-Santana ${ }^{1}$, T.E. Lázaro -Cantalejo ${ }^{2}$, Diego González- \\ González $^{3}$, Daniel Colinas Reyero ${ }^{3}$ \\ ${ }^{I}$ Department of Dermatology, Hospital Rio Hortega, Valladolid, Spain \\ ${ }^{2}$ Clínica Dermatológica Santa Teresa. Valladolid, Spain \\ ${ }^{3}$ Pathology Department, Hospital Rio Hortega, Valladolid, Spain
}

*Corresponding Author: $\boldsymbol{R}$ Giménez-García, Department of Dermatology, Hospital Rio Hortega, Valladolid, Spain,Email: rosagim@hotmail.com

\begin{abstract}
Background: Vulvar melanosis is a condition clinically characterized by pigmented lesions of irregular form and colour that may mimic malignant melanoma. Pigmented vulvar lesions are quite common and nearly $30 \%$ corresponded to melanosis. There are only a few published case reports and little is described about dermoscopic features of vulvar melanosis.
\end{abstract}

Case Reports: We report two cases of histologically confirmed vulvar melanosis. Dermoscopic examination revealed a ring like pattern (multiple round to ovoid structures arranged in a grape-like manner) and parallel pattern (linear or partially curved pigmentary streaks arranged in a parallel manner).

Conclusion: Dermoscopic analysis can improve accuracy in diagnosing pigmented vulvar skin lesions. Vulvar melanosis demonstrate different dermoscopic patterns, includindg rignlike, parallel, structurless or reticular-like differing from malignant melanoma.

Keywords: vulvar melanosis; vulvar hiperpigmentation; dermoscopy of vulvar melanosis.

\section{INTRODUCTION}

Vulvar melanosis (VM) is a condition clinically characterized by pigmented lesions of irregular form and colourthat may mimic malignant melanoma. Because pigmented lesions on mucous membranes and anogenital skin are atypical morphologically, they should be biopsied [1-3]. Roughly $10 \%$ of women will have a pigmented vulval lesion in her lifetime. [1,2] Even though vulvar melanosis has a benign prognosis, differential diagnosis should be considered including tumors such as basal cell carcinoma and melanoma where an early diagnosis is important $[1,2]$. There are only a few case reports about genital melanosisin literature and little is described about dermoscopic features of vulvarmelanosis [5-8]. We thought it would be interesting to present two clinical cases of recently attended patients with vulvar melanosis.

\subsection{Case 1}

JGL. 52-year-old woman with personal history of allergy to quinolones and intolerance to sulfonamides, hypertension and multiple sclerosis diagnosed in 1991 in treatment with betaferon, omeprazole, amlodipine, bromazepam, oxybutynin and solifenacin. Generalized vitiligo since she was 8 years old. She was referred by the gynecologist due to the presence of pigmented lesions in genital area. At clinical examination, they are observed some asymmetric hyper pigmented lesions, of multifocal patched appearance, with irregular borders and brownish color located on the vulva at the level of the major and minor labia and to a lesser extent in the introitus, without affecting the vagina (Fig. 1a). Dermoscopic examination a ring like pattern was revealed (Fig. 1b). The histopathological study of the lesions revealed an epidermis with no relevant alterations and normal presence of melanocytes in the basal layer, with a 
minimal lymphocytic inflammatory infiltrate and some melanophages in the underlying dermis (Fig. 1c). Patient is regularly monitored in the dermatology department.

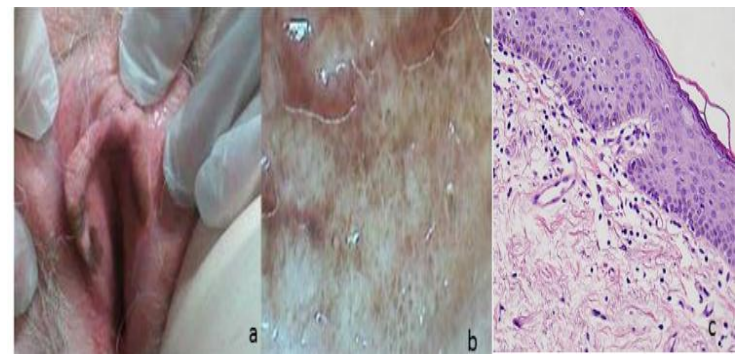

Fig1: a: Multifocal patched hiperpigmented lesions, with irregular borders and brownish color located on the major and minor labia; b: Dermoscopic analysis ringlike pattern; $c$ : histologically epidermis with no relevant alterations minimal lymphocytic inflammatory infiltrate and some melanophages in the underlying dermis

\subsection{Case 2}

35-year-old woman who, after the birth of her second child, is warned byher gynecologist that she had a spot on the genital area. The lesion is asymptomatic. At clinical examination they are observed some hyperpigmented slate-gray macules located bilaterally on the labia majora without defined borders (Fig. 2a). At dermatoscopic analysis a parallel pattern was shown in some areas (Fig. 2b). Histologically epithelial hyperplasia, elongationof the rete ridges, increasedmelanin in the basal layerwith slightly increase in melanocytic density, and stromal melanophages without atypia were found. No psoriasiform, spongiform or lichenoid signs were observed.These features were consistent with vulvar melanosis.

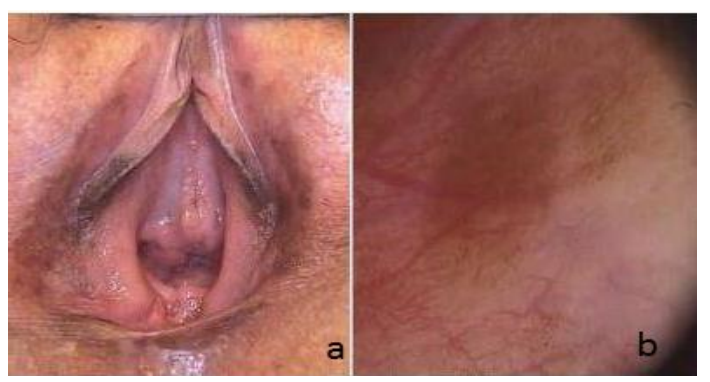

Fig2: a: Hyperpigmented slate-gray macules located bilaterally on the labia majora; $b$ : dermoscopically featuring a parallel pattern

\section{DISCUSSION}

The normal tone of the skin is a variable in the population, and therefore, we can find cases of physiological hyperpigmentation in people of dark complexion and in women under hormonal influence such as pregnancy or fertility treatments [3]. The presence of pigmented lesions has been demonstrated in $19 \%$ of a group of women studied in a specialized clinic, of which only $24 \%$ had noticed the presence of hyperpigmented vulvar lesions [7]. RongerSavle S et al. [8] studied 67 cases of pigmented lesions of the vulva, of which 20 (29\%) corresponded to melanosis.

The pathogenesis of vulvar melanosis is unknown. Clinically, vulvar melanosis presents as a single or more commonly multiple, asymmetric macules or patches, with tan-tobrown color, irregular borders, and variable size. In most cases, it develops on the labia minora, but it can also occur on the labia majora, vaginal introitus and perineum [2, 4]. Some authors have speculated about a correlation between vulvar melanosis and malignant melanoma. However, no increase in melanoma or squamous cell carcinomas has been seen in patients with vulvar melanosis [2, 4]. The diagnosis must be confirmed by histophatological study since the clinical characteristics may be similar to those of melanoma [5]. Histopathology of melanosis or vulvar lentiginosis is characterized by an increase in melanin in the basal layer, a discrete melanocytic hyperplasia without nest formation, as well as an epithelial hyperplasia and melanophages in the dermis. [3, 4]In our cases we did not find cellular atypia.

With respect to dermatoscopic study, different patterns present in vulvar melanosis have been described; structureless, parallel, reticular-like and annular, also known as ringlike pattern[5, $7,8]$. Ring like pattern was found in $32 \%$ of melanosis cases [5]. It is characterized by multiple round to oval structures, light color, with dark brown well-defined regular borders, arranged in a grape-like manner in some areas [5]. The homogeneous or structure less pattern also appears with some frequency and is histologically correlated with the presence of melanophages [7]. Parallel pattern is found more frequently in locations such as the labia and the penis. This can be differentiated from nevi and melanoma due to the absence of characteristic lesions (reticulum, globules, pigmented network) [7]. In some studies, this parallel pattern is the most frequently observed in epithelial hyperpigmentation without melanocytic hyperplasia [6]. Reflectance confocal microscopy can facilitate the diagnosis of pigmented lesions in the vulva. Papillae rimmed by 
bright monomorphus cells (a pattern that on the skin is typical of lentiginosis and that indicates a greater pigmentation of the keratinocytes of the basal layer of the mucosa) is a major feature associated with vulvar melanosis $[2,6]$.

The differential diagnosis of vulvar lesions is not always easy, and we must distinguish between some pigmented pathologies such as malignant melanoma, drug-induced skin pigmentation, nevus, acanthosis nigricans, vulvar intraepithelial neoplasia, Paget's disease and squamous cell carcinoma; and lesions that look pigmented but do not contain melanin, such as seborrheic keratosis, vascular lesions (angiomas and angiokeratomas) and comedones [3].

Vulvar melanosis occurs most often in premenopausal women, and when occurring in children, one of several syndromes with mucous membrane lentiginosis, and even in keratinized skin, should be considered. These include PeutzJeghers syndrome, LEOPARD syndrome, Bannayan - Riley - Ruvalcaba syndrome (Autosomal dominant disease that presents with a characteristic triad formed by genital lentiginosis, macrocephaly and intestinal polyposis), Dowling Degos disease, Carney syndrome and LAMB syndrome (Cardiocutaneous syndromes that include atrialmyxomas, blue nevi on the skin and genital mucosa, with papules or nodules on the skin and tongue), Beare-Stevenson syndrome and LaugierHunzinkersyndrome (rare acquired disorder characterized by diffuse hyperpigmentation of the oral mucosa and longitudinal melanonychia in adults) $[2,3]$. Therefore, it is important to rule out other mucocutaneous pigmentary disorders that require medical management, as well as a rapid clinical recognition that can avoid the need for excessive and invasive procedures and treatments.

Several associations between vulvar melanosis and other pathologies have been studied, such as melanoma, lichen sclerosus, taking oral contraceptives [2], immediate postpartum period [2], HPV infection and vilitigo.No increased risk of developing melanoma or squamous cell carcinoma has been observed in patients with vulvar melanosis [2, 4]. In literature, it has been only described a case of vulvar melanosis in a patient with bladder melanoma [4]. However, an increased risk of developing VM has been observed in cases of lichen sclerosus. [3]The melanosis has a benign course. Two follow-up studies of vulvar melanosis that were carried out during a period of time from 2 to 14 years, showed a minimum clinical change and an absence of malignancy [2].

\section{SPECIFIC}

Vulvar melanosis is quite common and may mimic malignant melanoma. Dermoscopic analysis can improve accuracy in diagnosing pigmented vulvar skin lesions

\section{Teaching Points}

- Vulvar melanosis is quite common and may mimic malignant melanoma.

- The differential diagnosis of vulvar lesions is not always easy, and we must distinguish between some pigmented pathologies.

- Dermoscopic analysis can improve accuracy in diagnosing pigmented vulvar skin lesions. Vulvar melanosis demonstraterignlike, parallel, structurless or reticular-like dermoscopic patterns differing from malignant melanoma.

- The diagnosis must be confirmed by histophatological study. Histopathology of melanosis or vulvar lentiginosis is characterized by an increase in melanin in the basal layer, a discrete melanocytic hyperplasia without nest formation, as well as an epithelial hyperplasia and melanophages in the dermis.

\section{REFERENCES}

[1] Nellar MFCurmonaMC, Garay IS, Kurpis M, Ruiz Lascano A. Lesiones pigmentadas de la vulva. Arch. Argent. Dermatol. 2013; 63: 137142.

[2] Murzaku EC, Penn LA, Hale CS, Pomeranz MK, Polsky D.Vulvar nevi, melanosis, and melanoma: an epidemiologic, clinical, and histopathologic review. J Am AcadDermatol. 2014; 71:1241-9.

[3] Edwards, L. Pigmented vulvar lesions. Dermatol Therapy. 2010; 23:449-457.

[4] Venkatesan A. Pigmented lesions of the vulva. Dermatol Clin. 2010; 28:795-805.

[5] Ferrari A, Buccini P, Covello R, De Simone P, Silipo V, Mariani G, Eibenschutz L, Mariani L, Catricalà $\mathrm{C}$. The ringlike pattern in vulvar melanosis: a new dermoscopic clue for diagnosis. Arch Dermatol. 2008;144:1030-4

[6] Cinotti E, Perrot JL, Labeille B, Adegbidi H, Cambazard F. Reflectance Confocal Microscopy for the diagnosis ovulvar melanoma and melanosis: preliminary results. Dermatol Surg. 2012; 38: 1962-1967. 
[7] Mannone F, De Giorgi V, Cattaneo A, Massi D, De Magnis A, Carli P. Dermoscopic features of mucosal melanosis. Dermatol Surg. 2004; 30: 1118-1123.
[8] Ronger-Savle S, Julien V, Duru G, Raudrant D, Dalle S, Thomas L. Features of pigmented vulval lesions on dermoscopy. Br J Dermatol. 2010; 164: 54-61.

Citation: R Giménez-García, Cintia Gonzalez-Santana, T.E. Lázaro-Cantalejo, Diego González-González, Daniel Colinas Reyero.Vulvar Melanosis: Two Case Report. ARC Journal of Dermatology. 2018; 3(2):4-7. doi:dx.doi.org/10.20431/2456-0022.0302002.

Copyright:(C) 2018 Authors. This is an open-access article distributed under the terms of the Creative Commons Attribution License, which permits unrestricted use, distribution, and reproduction in any medium, provided the original author and source are credited. 